Supplement of Biogeosciences, 18, 2449-2463, 2021 https://doi.org/10.5194/bg-18-2449-2021-supplement (c) Author(s) 2021. CC BY 4.0 License.

(c) (1)

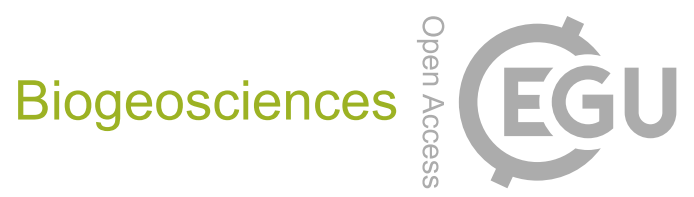

Supplement of

\title{
Biogeochemical and plant trait mechanisms drive enhanced methane emis- sions in response to whole-ecosystem warming
}

Genevieve L. Noyce and J. Patrick Megonigal

Correspondence to: Genevieve L. Noyce (noyceg@ si.edu)

The copyright of individual parts of the supplement might differ from the article licence. 
Table S1. Calculated limit of detection ( $\mathrm{LOD}_{\text {flux }}$ ) of chamber flux system.

\begin{tabular}{cc}
\hline $\begin{array}{c}\text { \# of stacked } \\
\text { chambers }\end{array}$ & $\begin{array}{c}\mathrm{LOD}_{\text {flux }} \\
\left(\mu \mathrm{mol} \mathrm{CH} \mathrm{m}^{-2} \mathrm{~d}^{-1}\right)\end{array}$ \\
\hline 1 & 8.7 \\
2 & 17.4 \\
3 & 26.1 \\
4 & 34.9 \\
\hline
\end{tabular}


Table S2. Estimated annual $\mathrm{CH}_{4}$ flux for each plot in 2017, 2108, and 2019.

\begin{tabular}{|c|c|c|c|c|c|c|c|c|c|}
\hline \multirow[t]{2}{*}{ Plot } & \multirow{2}{*}{$\begin{array}{c}\text { Temperature } \\
\text { Treatment }\end{array}$} & \multirow{2}{*}{$\begin{array}{c}\text { Plant } \\
\text { Community }\end{array}$} & \multirow{2}{*}{$\begin{array}{c}\text { Replicate } \\
\text { Transect }\end{array}$} & \multicolumn{3}{|c|}{$\begin{array}{l}\text { Estimated Annual } \mathrm{CH}_{4} \text { Flux } \\
\qquad\left(\mathrm{mmol} \mathrm{CH} \mathrm{CH}_{4}^{-2} \mathrm{yr}^{-1}\right)\end{array}$} & \multicolumn{3}{|c|}{$\begin{array}{l}\text { Mean (SE) Annual } \mathrm{CH}_{4} \text { Flux } \\
\left(\mathrm{mmol} \mathrm{CH}_{4} \mathrm{~m}^{-2} \mathrm{yr}^{-1}\right)\end{array}$} \\
\hline & & & & 2017 & 2018 & 2019 & 2017 & 2018 & 2019 \\
\hline 310 & \multirow{3}{*}{ Ambient } & \multirow{3}{*}{$\mathrm{C}_{3}$} & 1 & 59.9 & 125.5 & 83.7 & \multirow{3}{*}{$\begin{array}{l}58.4 \\
(6.9)\end{array}$} & \multirow{3}{*}{$\begin{array}{l}161.4 \\
(37.0)\end{array}$} & \multirow{3}{*}{$\begin{array}{c}80.4 \\
(11.4)\end{array}$} \\
\hline 320 & & & 2 & 69.6 & 235.4 & 98.3 & & & \\
\hline 330 & & & 3 & 45.8 & 123.2 & 59.1 & & & \\
\hline 311 & \multirow{3}{*}{$+1.7^{\circ} \mathrm{C}$} & \multirow{3}{*}{$\mathrm{C}_{3}$} & 1 & 58.8 & 186.6 & 82.8 & \multirow{3}{*}{$\begin{array}{c}69.2 \\
(21.8)\end{array}$} & \multirow{3}{*}{$\begin{array}{l}189.2 \\
(23.0)\end{array}$} & \multirow{3}{*}{$\begin{array}{l}82.1 \\
(6.5)\end{array}$} \\
\hline 321 & & & 2 & 111.0 & 230.2 & 93.0 & & & \\
\hline 331 & & & 3 & 37.8 & 150.9 & 70.6 & & & \\
\hline 312 & \multirow{3}{*}{$+3.4{ }^{\circ} \mathrm{C}$} & \multirow{3}{*}{$\mathrm{C}_{3}$} & 1 & 87.5 & 160.5 & 113.6 & \multirow{3}{*}{$\begin{array}{c}82.6 \\
(12.2)\end{array}$} & \multirow{3}{*}{$\begin{array}{l}178.8 \\
(21.5)\end{array}$} & \multirow{3}{*}{$\begin{array}{c}90.7 \\
(13.4)\end{array}$} \\
\hline 322 & & & 2 & 100.9 & 221.7 & 67.2 & & & \\
\hline 332 & & & 3 & 59.5 & 154.1 & 91.2 & & & \\
\hline 313 & \multirow{3}{*}{$+5.1{ }^{\circ} \mathrm{C}$} & \multirow{3}{*}{$\mathrm{C}_{3}$} & 1 & 334.4 & 621.9 & 665.1 & \multirow{3}{*}{$\begin{array}{l}178.0 \\
(78.2)\end{array}$} & \multirow{3}{*}{$\begin{array}{c}343.2 \\
(140.0)\end{array}$} & \multirow{3}{*}{$\begin{array}{r}317.6 \\
(174.5)\end{array}$} \\
\hline 323 & & & 2 & 101.4 & 180.1 & 115.6 & & & \\
\hline 333 & & & 3 & 98.3 & 227.6 & 172.1 & & & \\
\hline 440 & \multirow{3}{*}{ Ambient } & \multirow{3}{*}{$\mathrm{C}_{4}$} & 4 & 63.1 & 424.5 & 121.2 & \multirow{3}{*}{$\begin{array}{l}55.0 \\
(6.9)\end{array}$} & \multirow{3}{*}{$\begin{array}{l}425.5 \\
(88.6)\end{array}$} & \multirow{3}{*}{$\begin{array}{l}205.1 \\
(45.2)\end{array}$} \\
\hline 450 & & & 5 & 60.8 & 579.4 & 217.8 & & & \\
\hline 460 & & & 6 & 41.2 & 272.4 & 276.3 & & & \\
\hline 441 & & & 4 & 37.6 & 298.4 & 154.9 & & & \\
\hline 451 & $+1.7^{\circ} \mathrm{C}$ & $\mathrm{C}_{4}$ & 5 & 69.7 & 518.3 & 695.0 & $\begin{array}{c}61.7 \\
(12.3)\end{array}$ & $\begin{array}{l}370.9 \\
(73.7)\end{array}$ & $\begin{array}{c}417.6 \\
(1561)\end{array}$ \\
\hline 461 & & & 6 & 77.9 & 295.9 & 403.0 & & & \\
\hline 442 & & & 4 & 40.1 & 301.0 & 173.5 & & & \\
\hline 452 & $+3.4{ }^{\circ} \mathrm{C}$ & $\mathrm{C}_{4}$ & 5 & 89.8 & 636.5 & 614.8 & $\begin{array}{c}84.8 \\
(24.5)\end{array}$ & $\begin{array}{l}471.0 \\
(96.9)\end{array}$ & $\begin{array}{c}386.8 \\
(127.6)\end{array}$ \\
\hline 462 & & & 6 & 124.5 & 475.3 & 371.9 & & & \\
\hline 443 & & & 4 & 84.3 & 288.8 & 127.7 & & & \\
\hline 453 & $+5.1^{\circ} \mathrm{C}$ & $\mathrm{C}_{4}$ & 5 & 185.2 & 1425.7 & 706.6 & $\begin{array}{l}157.8 \\
(371)\end{array}$ & $\begin{array}{c}879.3 \\
(328.9)\end{array}$ & $\begin{array}{c}469.9 \\
(1752)\end{array}$ \\
\hline 463 & & & 6 & 203.8 & 923.3 & 575.5 & & & \\
\hline
\end{tabular}




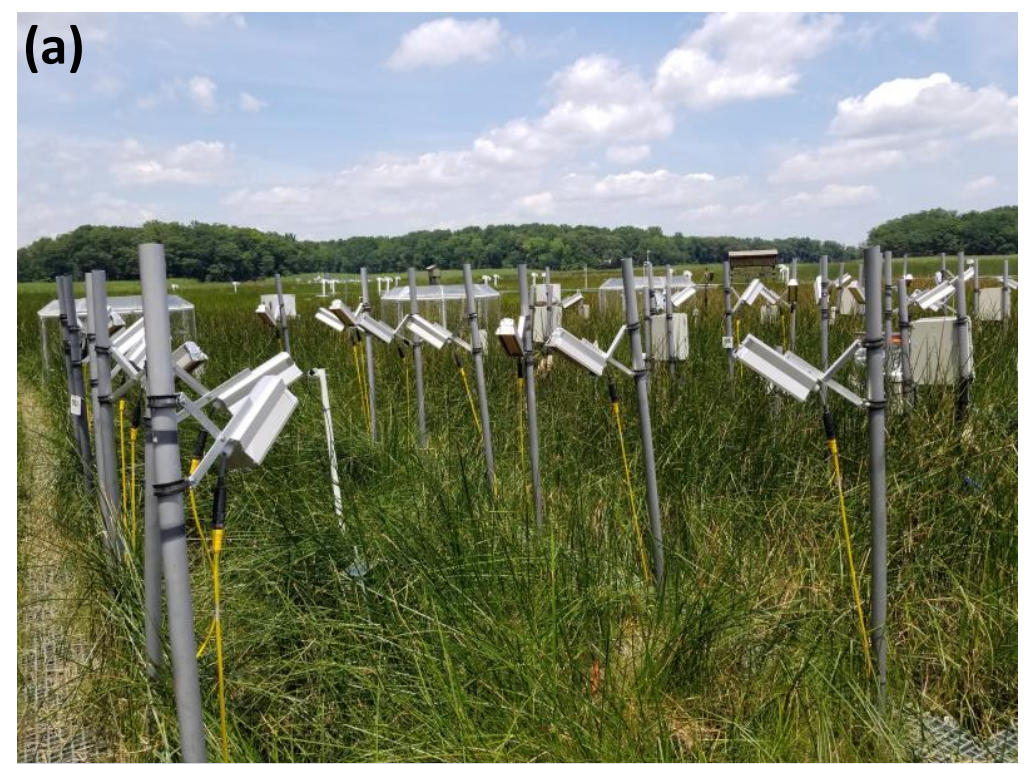

(b)

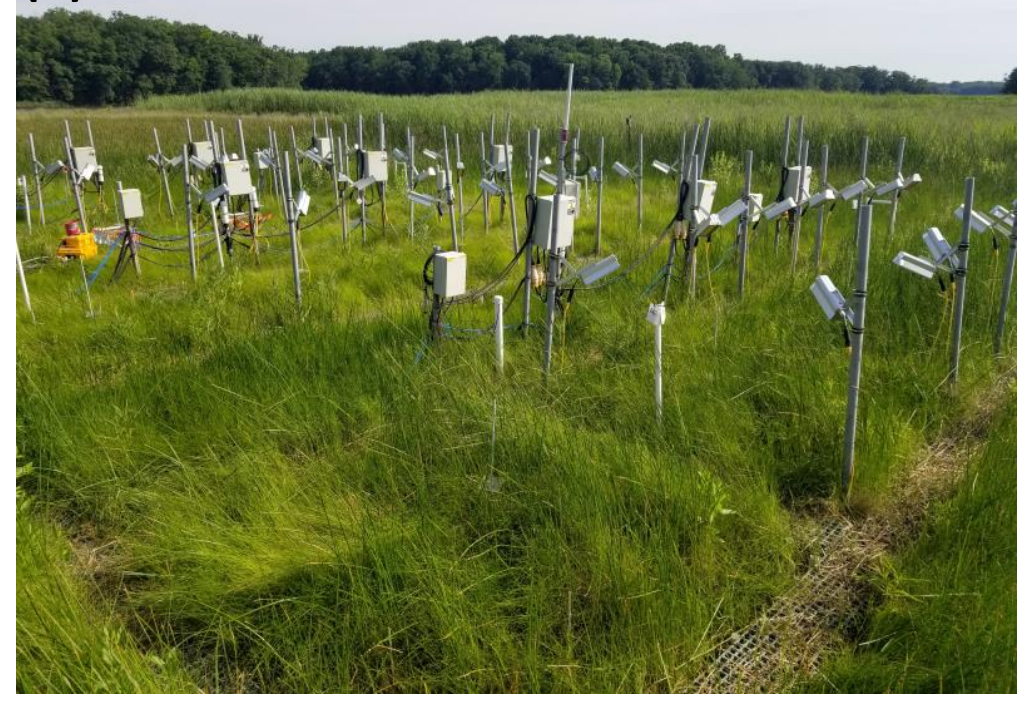

Figure S1. SMARTX plots in (a) the $\mathrm{C}_{3}$ community (dominated by Schoenoplectus americanus) and (b) the $\mathrm{C}_{4}$ community (dominated by Spartina patens and Distichlis spicata). Photos taken by G. Noyce. 


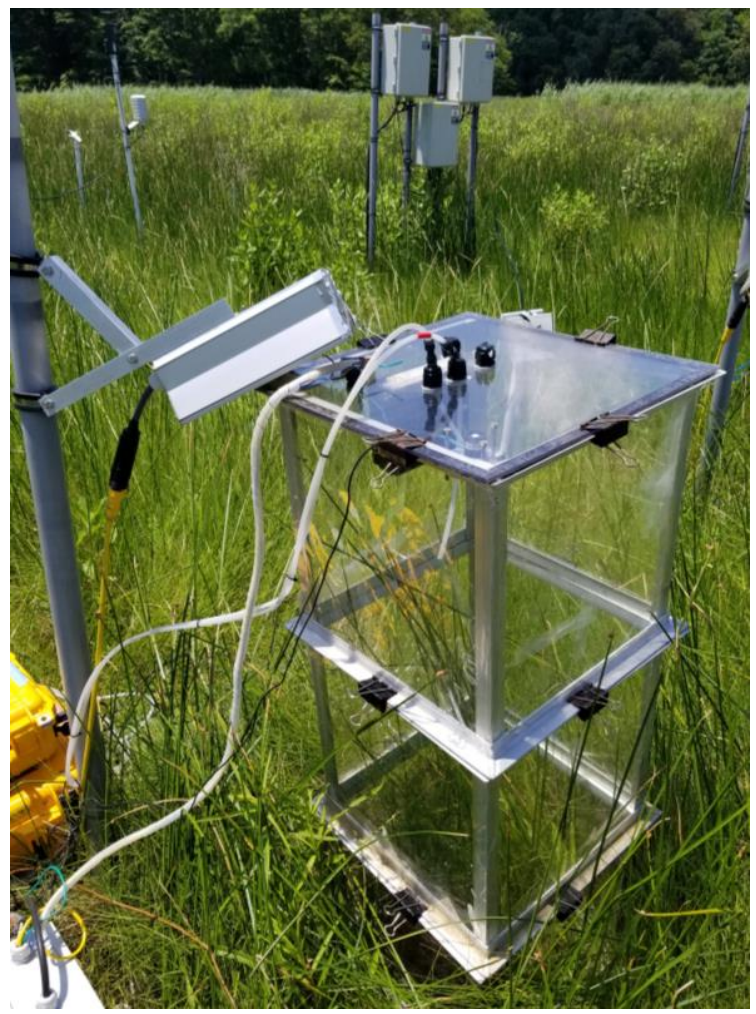

Figure S2. Stacked modular flux chambers. Photo taken by G. Noyce.

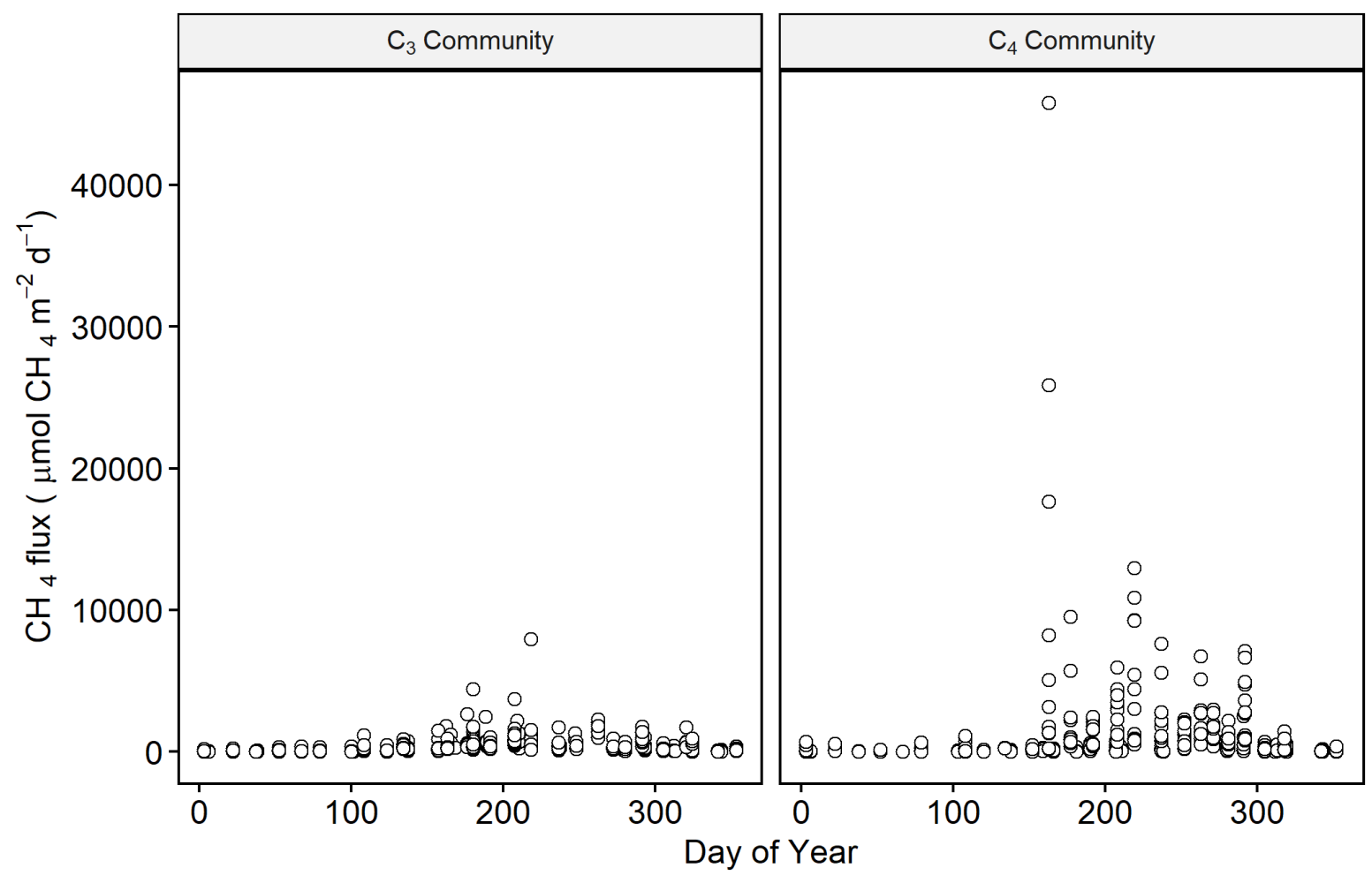

Figure S3. All raw $\mathrm{CH}_{4}$ flux data. 


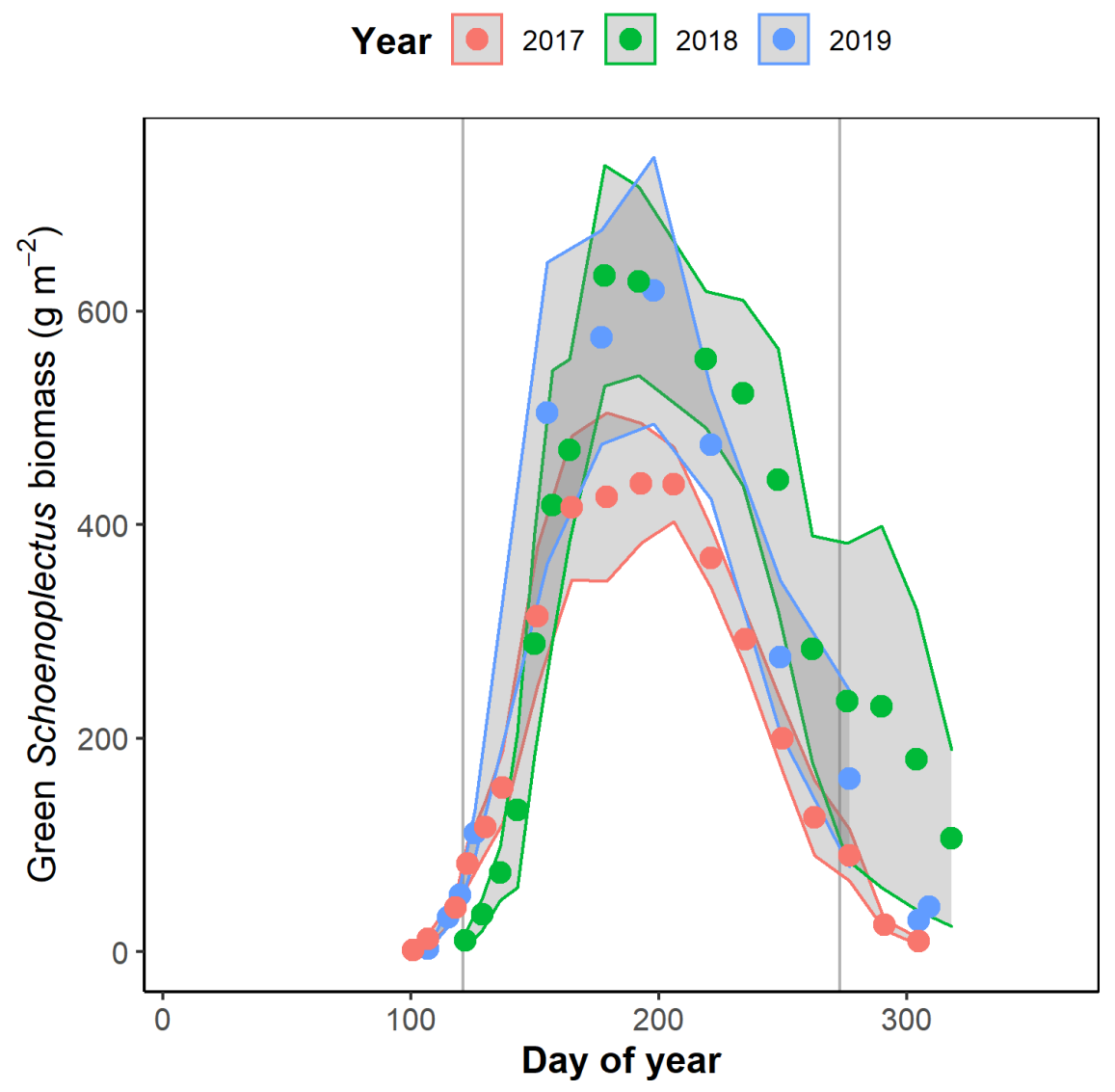

Figure S4. Schoenoplectus phenological data for 2017 - 2019 in ambient temperature plots. Points indicate means $(n=3)$ and shaded area indicates SE. The vertical lines indicate the 'growing season', e.g. May 1 through Sep 30. 


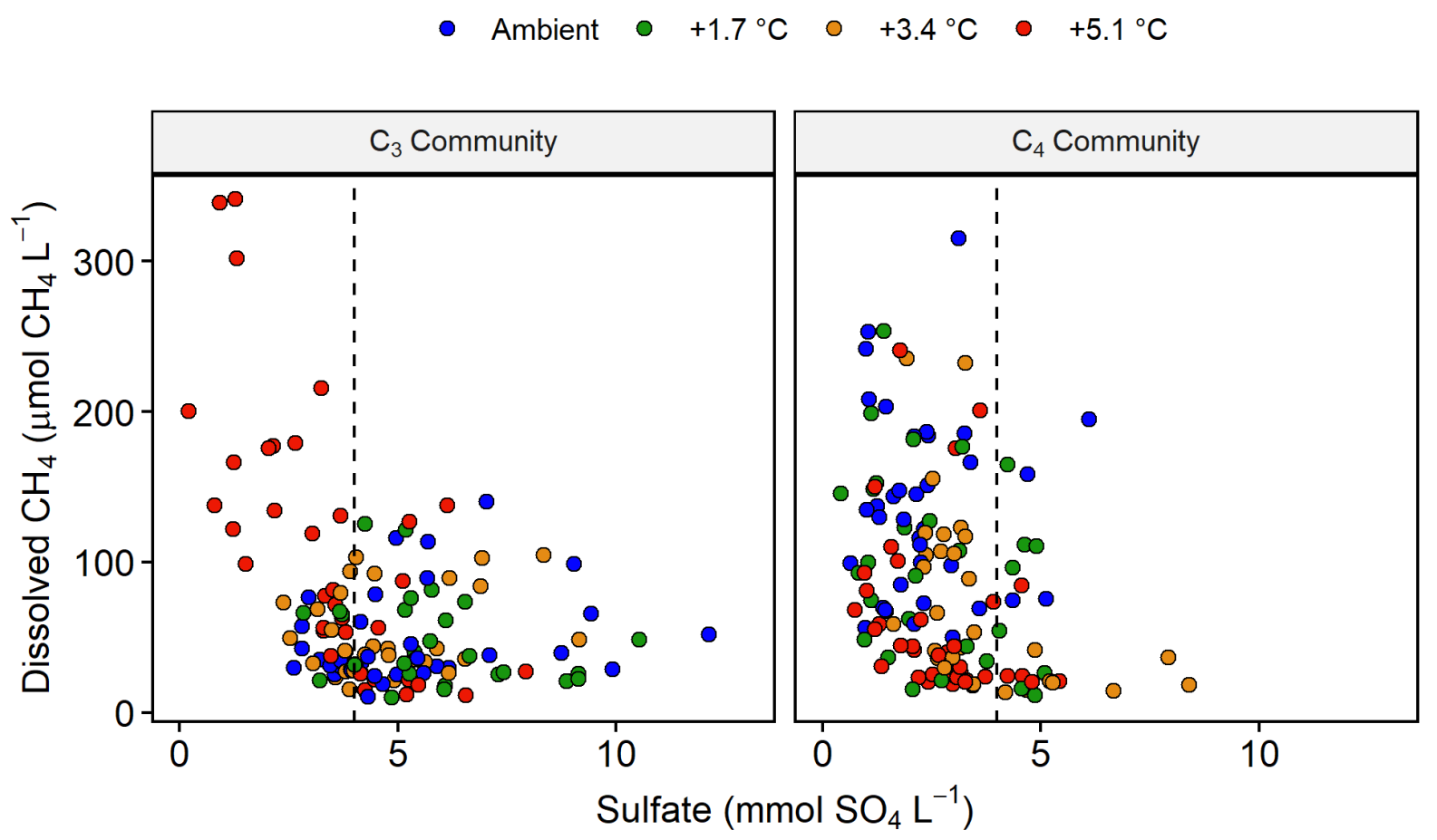

Figure S5. Mean concentrations of $\mathrm{CH}_{4}$ and $\mathrm{SO}_{4}$ for each plot and month that porewater was sampled. Colors indicate temperature treatment. The vertical dashed line indicates the $4 \mathrm{~mm}\left[\mathrm{SO}_{4}\right]$ threshold below which acetoclastic and hydrogenotrophic methanogenesis are released from substrate competition. 


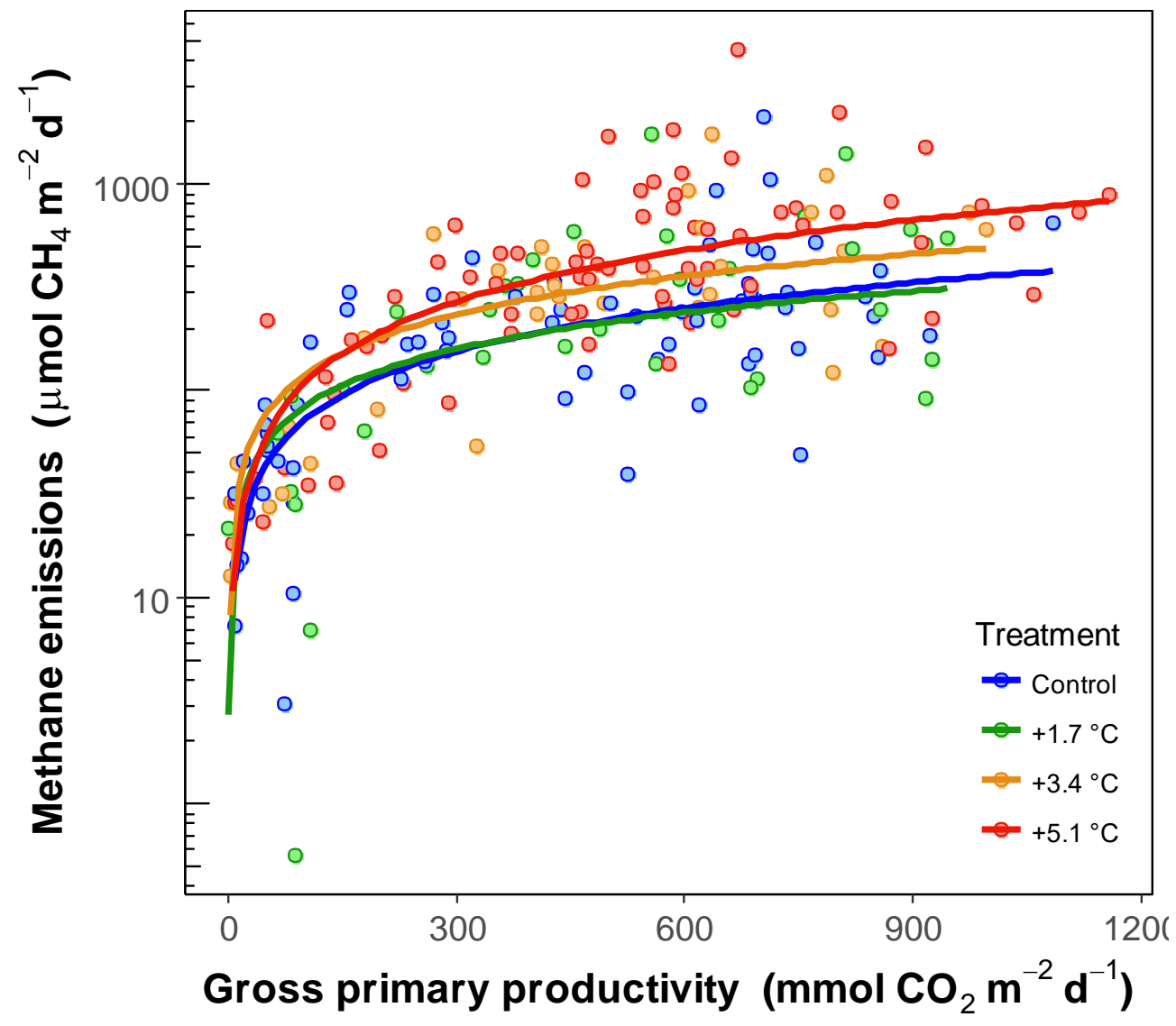

Figure S6. Methane emissions compared to gross primary productivity measured at the same time. $\mathrm{CH}_{4}$ emissions were strongly correlated with GPP. 

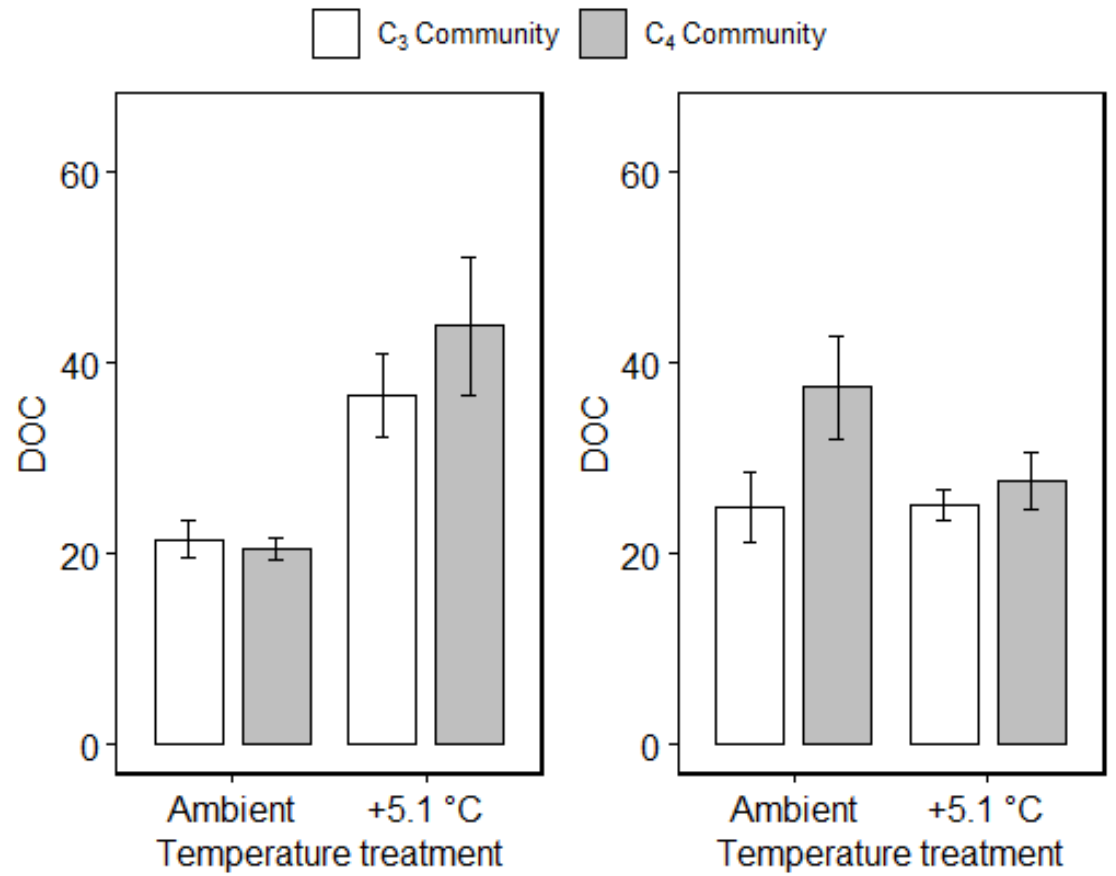

Figure S7. Comparison of DOC in July 2019 from the $\mathrm{C}_{3}$ community dominated by Schoenoplectus (open bars) and the $\mathrm{C}_{4}$ community dominated by Spartina and Distichlis (grey bars). Left: In the dominant rooting zone $(10-20 \mathrm{~cm})$; Right: below the rooting zone $(40-120 \mathrm{~cm})$. Error bars indicate SE. 